\title{
The Effects of National Health Insurance Denture Coverage Policies for the Elderly on the Unmet Dental Needs of the Edentulous Elderly
}

\author{
Eunsuk Ahn, Ji-Min Hwang ${ }^{1}$, and Ji-Hyoung $\mathrm{Han}^{2, \dagger}$ \\ Department of Dental Hygiene, Kyungbok University, Pocheon 11138, \\ ${ }^{1}$ Department of Dental Hygiene, Baekseok Culture University, Cheonan 31065 , \\ ${ }^{2}$ Department of Dental Hygiene, Suwon Science College, Hwaseong 18516, Korea
}

\begin{abstract}
As the elderly population increases, they are increasingly affected by oral health problems. Therefore, efforts are being made to improve the oral health of older people, alleviate mental discomfort, and reduce unmet dental needs. This study was conducted to confirm the relationship between the National Health Insurance Elderly Denture Coverage and the unmet dental need for the edentulous elderly, as part of the protection policy. We analyzed the 2011 and 2013 Community Health Survey data of the edentulous elderly, aged 75 years or older, before 2012. In order to more precisely confirm the effects of the denture donation policy on unmet dental care, basic life recipients who were subject to the free elderly prosthetic project were excluded from the analysis. The final analysis included 20,400 subjects. According to our investigation of the factors that affect the unmet dental needs of the elderly, the National Health Insurance Elderly Denture Coverage did not affect unmet dental needs. The statistically significant variables that affected the unmet dental needs of the elderly were education and income levels, which are representative socioeconomic status variables. The lower the level of education, the unhealthier the dental care experience, and income levels showed a similar tendency. The elderly who have a low socioeconomic status are more likely to experience unmet dental needs because they lack the knowledge and socioeconomic ability to pay for dental care. Therefore, the policy for health protection of the entire elderly population should be continuously expanded. In addition, the socioeconomically vulnerable groups may have health problems due to the restriction of medical use, which may lead to quality of life deterioration.
\end{abstract}

Key Words: Dentures, Elderly, National Health Insurance Coverage, Unmet need

\section{Introduction}

To provide adequate health care services, South Korea implemented a national health insurance system for all its citizens, starting from 1989. However, high out-of-pocket cost due to limited benefits from low contribution-low coverage policies represents a barrier to the utilization of health care services ${ }^{1}$. In other words, mandatory copayments and out-of-pocket costs for non-coverage items act as key factors in the utilization of services for meeting the health care needs of individuals. This also makes it more difficult to meet the health care needs of the socioeconomically vulnerable population ${ }^{1,2)}$. The state of not meeting health care needs or wants is referred to as unmet health care needs, which also implies that for patients with certain diseases, the illness could have been prevented or eliminated if health care services had been properly utilized. Studies on unmet health care needs have mostly been used to identify problems associated with health care service accessibility ${ }^{3,4)}$.

Aging has become a more serious issue with the rapidly growing elderly population; and as a result, elderly health has emerged as important social issue ${ }^{2,5}$. In particular, oral health is closely associated with weakened tooth function 
and high rates of tooth loss, with the elderly being relatively more vulnerable than other age groups ${ }^{6-8)}$. However, because of financial vulnerability during the elderly years, proper dental services with expensive dental prostheses are being neglected ${ }^{5,7-9)}$. In response, the Korea Ministry of Health and Welfare implemented a free dental prostheses program for elderly recipients of the basic livelihood security benefit, as an effort towards facilitating the recovery of oral functions in the elderly. However, this program benefits only the elderly who are recipients of the basic livelihood security benefit, which still leaves the medical cost burden of dental prostheses on the lowincome elderly population who are not recipients of the basic livelihood security benefit. To address this issue, a National Health Insurance (NHI) coverage policy for complete dentures for the elderly who are 75 years and older was implemented in July 2012, as a part of the NHI coverage expansion policy ${ }^{10)}$. The present study aimed to investigate the effects of the NHI complete denture coverage policy for the elderly on the unmet dental needs of the edentulous elderly, aged 75 years and older, relative to before and after 2012, when the policy was implemented. The study also aimed to use the findings to provide basic data for the development of future policies that can help improve oral health care accessibility for the elderly.

\section{Materials and Methods}

\section{Subjects}

The present study analyzed data from the Community Health Survey (CHS). The CHS is a survey that is conducted for the purpose of establishing the basis for evidence-based health care programs by collecting community level health statistics. The survey measures health behaviors (smoking, drinking, and physical activity), oral health status, health care utilization, and quality of life in adults aged 19 years and older ${ }^{11)}$. The study analyzed 2011 and 2013 CHS data, since the NHI coverage policy on complete dentures for the elderly was implemented in 2012. To include only completely edentulous subjects who were the beneficiaries of NHI coverage for dentures for the elderly, only the elderly who were 75 years of age and older, who responded as having a maxillary or mandibular edentulous state on the question of current missing teeth status, were included in the study. For a more accurate analysis of the effects of NHI coverage policy on dentures for the elderly on unmet health care needs, recipients of basic livelihood security benefits who qualify for the free dental prostheses program were excluded. As a result, 20,400 people were included in the final analysis. Before starting the study, approval was obtained from the Institutional Review Board at Suwon Science College (IRB2-7008167-AB-N-01-201803-HR-001-01).

\section{Variables used in the analysis}

The dependent variable used in the analysis was the experience of unmet dental care needs, which was determined based on the survey question, "Did you feel a need for dental treatment but did not receive the necessary care during the past one year?" The major explanatory variable, NHI coverage of dentures for the elderly, was generated by dividing the subjects who were surveyed in 2011 as pre-implementation and in 2013 as postimplementation. The other explanatory variables included sex, age, education level, income level, basic livelihood security benefit, and residence.

\section{Analysis method}

A basic analysis was performed to examine the data

Table 1. General Characteristics

\begin{tabular}{llc}
\hline & Classification & Data \\
\hline Sex & Male & $7,141(35.00)$ \\
& Female & $13,259(65.00)$ \\
Age (y) & & $80.47 \pm 4.52$ \\
Region & City & $5,337(26.16)$ \\
& Rural & $15,063(73.84)$ \\
Education level & Below elementary school & $17,367(85.18)$ \\
& Middle/high school & $2,500(12.25)$ \\
& Above college & $522(2.56)$ \\
Marriage status & Having spouse & $9,047(44.52)$ \\
& Not having spouse & $11,274(55.48)$ \\
Income level & Lower & $7,666(40.11)$ \\
& Middle & $5,670(29.67)$ \\
& Higher & $5,776(30.22)$ \\
Private insurance & Yes & $1,477(7.30)$ \\
& No & $18,751(92.70)$ \\
\hline
\end{tabular}

Values are presented as number $(\%)$ or mean \pm standard deviation. 
characteristics, while a chi-squared test was performed to identify the differences in unmet dental needs according to NHI coverage of dentures for the elderly. Lastly, a logistic regression analysis was performed to identify the factors influencing unmet dental needs. All analyses were performed using STATA ver. 11.0 (Stata Corp., College Station, TX, USA) with significance level set to $\mathrm{p}=0.05$.

\section{Results}

\section{General characteristics}

The general characteristics of the subjects are shown in Table 1. Among the edentulous elderly subjects, there were more females $(65.00 \%)$ than males $(35.00 \%)$, and their mean age was 80.47 years. There was a higher percentage of elderly living in rural areas $(73.84 \%)$ than in cities $(26.16 \%)$, while below elementary school $(85.18 \%)$ was the most common response for education level. With respect to marital status, $55.48 \%$ did not have a spouse, while "lower" (40.11\%) was the most common response for income level. Meanwhile, the majority of the edentulous elderly subjects $(92.70 \%)$ did not have private insurance.

\section{Comparison of unmet dental needs in the edentulous elderly according to $\mathrm{NHI}$ coverage of dentures for the elderly \\ Table 2 shows the results of differences in unmet dental} needs of the edentulous elderly between before and after implementation of NHI coverage of dentures for the elderly. The percentage of elderly who experienced unmet dental needs was very similar before and after implementation of NHI coverage, with rates of $20.29 \%$ and $20.34 \%$, respectively. With respect to differences in the experience of unmet dental needs according to general characteristics, for area of residence, those who reside in rural areas showed an increase of $1.03 \%$, from $73.77 \%$ to $74.80 \%$, from before to after the implementation of coverage, respectively $(\mathrm{p}<$ 0.05). With respect income level, the lower income level group showed a decreasing trend, from $38.92 \%$ to $37.93 \%$, the middle-income level group showed a slightly increasing trend, and the higher income level group showed almost no difference $(\mathrm{p}<0.05)$.

Table 2. Comparison of Unmet Dental Need according to Elderly Denture Coverage

\begin{tabular}{|c|c|c|c|c|c|c|}
\hline & \multicolumn{3}{|c|}{ Before elderly denture coverage } & \multicolumn{3}{|c|}{ After elderly denture coverage } \\
\hline & $\begin{array}{l}\text { Non experience } \\
\text { unmet dental need }\end{array}$ & $\begin{array}{c}\text { Experience } \\
\text { unmet dental need }\end{array}$ & p-value & $\begin{array}{l}\text { Non experience } \\
\text { unmet dental need }\end{array}$ & $\begin{array}{c}\text { Experience } \\
\text { unmet dental need }\end{array}$ & $\mathrm{p}$-value \\
\hline \multicolumn{7}{|l|}{ Sex } \\
\hline Male & $2,748(35.64)$ & $630(32.09)$ & 8.668 & $3,046(35.72)$ & $712(32.69)$ & 6.979 \\
\hline Female & $4,962(64.36)$ & $1,333(67.91)$ & 0.003 & $5,482(64.28)$ & $1,466(67.31)$ & 0.008 \\
\hline \multicolumn{7}{|l|}{ Region } \\
\hline City & $2,022(26.23)$ & $558(28.43)$ & 3.873 & $2,149(25.20)$ & $603(27.69)$ & 5.617 \\
\hline Rural & $5,688(73.77)$ & $1,405(71.57)$ & 0.049 & $6,379(74.80)$ & $1,575(72.31)$ & 0.018 \\
\hline \multicolumn{7}{|l|}{ Education level } \\
\hline Below elementary school & $6,582(85.44)$ & $1,735(88.39)$ & 13.614 & $7,145(83.83)$ & $1,888(86.69)$ & 13.302 \\
\hline Middle/high school & $903(11.72)$ & $194(9.88)$ & $<0.001$ & $1,145(13.43)$ & $254(11.66)$ & $<0.001$ \\
\hline Above college & $219(2.84)$ & $34(1.73)$ & & $233(2.73)$ & $36(1.65)$ & \\
\hline \multicolumn{7}{|l|}{ Marriage status } \\
\hline Having spouse & $3,398(44.24)$ & $824(42.17)$ & 2.709 & $3,895(45.86)$ & $923(42.50)$ & 7.912 \\
\hline Not having spouse & $4,283(55.76)$ & $1,130(57.83)$ & 0.100 & $4,598(54.14)$ & $1,249(57.50)$ & 0.005 \\
\hline \multicolumn{7}{|l|}{ Income level } \\
\hline Lower & $2,715(38.92)$ & $874(48.45)$ & 63.718 & $3,113(37.93)$ & $956(45.37)$ & 39.592 \\
\hline Middle & $2,054(29.44)$ & $504(27.94)$ & $<0.001$ & $2,523(30.74)$ & $586(27.81)$ & $<0.001$ \\
\hline Higher & $2,207(31.64)$ & $426(23.61)$ & & $2,572(31.34)$ & $565(26.82)$ & \\
\hline \multicolumn{7}{|l|}{ Private insurance } \\
\hline Yes & $581(7.62)$ & $112(5.77)$ & 7.883 & $647(7.63)$ & $135(6.23)$ & 4.935 \\
\hline No & $7,043(92.38)$ & $1,829(94.23)$ & 0.005 & 7,835 (92.37) & $2,031(93.77)$ & 0.026 \\
\hline
\end{tabular}

The p-values were analyzed by chi-squared test. 


\section{Factors influencing the unmet dental needs of the edentulous elderly}

The investigation of the factors that influence the unmet dental needs of the edentulous elderly showed that the group with below elementary school education level had higher experiences of unmet dental needs than the groups with higher education level $(\mathrm{p}<0.05)$. With respect to income level, the lower income level group had a higher experience of unmet dental needs than the middle-income level group $(\mathrm{p}<0.05$, Table 3$)$.

\section{Discussion}

The basic idea behind health care is based on social justice, and the goal of health care is to provide quality medical services to recipients using methods that are accessible and affordable for everyone ${ }^{12)}$. Starting in July 2012, the South Korean government applied the expansion

Table 3. Factors Affecting Unmet Dental Need

\begin{tabular}{lccc}
\hline & OR & SE & $95 \%$ CI \\
\hline Sex & & & \\
Male & 1 & & \\
Female & 0.873 & 0.070 & $0.747 \sim 1.021$ \\
Age & 1.011 & 0.007 & $0.997 \sim 1.024$ \\
Region & & & \\
City & 1 & & \\
$\quad$ Rural & 0.898 & 0.063 & $0.783 \sim 1.031$ \\
Education level & & & \\
Below elementary school & 1 & & \\
Middle/high school & 0.821 & 0.079 & $0.681 \sim 0.990$ \\
Above college & 0.685 & 0.118 & $0.488 \sim 0.959$ \\
Marriage status & & & \\
Having spouse & 1 & & \\
$\quad$ Not having spouse & 0.968 & 0.074 & $0.834 \sim 1.125$ \\
Income level & & & \\
Lower & 1 & & \\
Middle & 0.835 & 0.062 & $0.722 \sim 0.967$ \\
Higher & 0.871 & 0.028 & $0.753 \sim 1.007$ \\
Private insurance & & & \\
Yes & 1 & & \\
$\quad$ No & 1.027 & 0.120 & $0.817 \sim 1.292$ \\
National health insurance coverage & & \\
Before coverage & 1 & & \\
After coverage & 1.027 & 0.062 & $0.912 \sim 1.157$ \\
\hline
\end{tabular}

Data was analyzed by logistic regression.

OR: odds ratio, SE: standard error, 95\% CI: 95\% confidence interval. of NHI coverage for resin complete dentures for the elderly, aged 75 years and older, to relieve the chewing difficulties that are faced by the elderly population, reduce their unmet health care needs, and promote improved oral health among the elderly ${ }^{13}$. To identify the effects of the NHI denture coverage policy for the elderly, the present study examined the effects of the policy on the unmet dental needs of the edentulous elderly, who are 75 years of age or older.

Although NHI coverage policies have achieved some success from the perspective of vertical equity improvement, there remains room for improvement with respect to horizontal equity. This can be interpreted in association with the influences of other factors. According to the logistic regression analysis results, even after adjusting for NHI coverage of dentures for the elderly and other factors, education and income levels were identified as the factors that have statistically significant influences on the unmet dental needs of the edentulous elderly. With respect to education level, the group with below elementary school education had a relatively higher experience of unmet dental needs than the groups with higher education levels, while a similar tendency was found with income level. Education and income levels are variables that represent socioeconomic inequality among health determining factors $^{5,9,12)}$. Education level is significant in that it reflects a person's socioeconomic status and also determines the socioeconomic inequality that a person may face during his or her lifetime ${ }^{13,14)}$.

As in the present study, previous studies have also reported that medical costs were a greater burden for those with lower household incomes; and as a result, unmet dental needs increase due to the greater medical cost burden that is associated with using dental care facilities ${ }^{6,7,15)}$. Musacchio et al. ${ }^{13)}$ reported that oral diseases appear unequally in those with low socioeconomic status or poor health status or function, while having a lower socioeconomic status increases the risk factors of oral health ${ }^{14,16)}$. In summarizing these results, elderly individuals with lower socioeconomic statuses define a population with health vulnerabilities, which increases their likelihood of experiencing health inequity due to a lack of knowledge about and the socioeconomic ability to 
pay for oral health care. Instead of policies that are applied uniformly to the entire elderly population, it is necessary to divide the population into specific groups and provide active intervention for the vulnerable population. Moreover, since the elderly population will continue to grow, long-term oral health issues from the perspective of welfare for the elderly should be handled, rather than policies that are limited to one-time service benefits. In particular, a prostheses program, which accounts for one of the most important aspect of oral health issues among the elderly, should be planned and implemented more effectively. Furthermore, a detailed strategy for comprehensive oral health programs that target the elderly should be established, as a preventive measure for reducing the need for dentures.

Because the present study was a cross-sectional study that only analyzed data from the 2011 and 2013 CHS, it is difficult to generalize our findings. Moreover, although the unmet health care needs of the edentulous elderly were survey by estimating the demand for dentures, the results may reflect a personal subjective assessment, due to a lack of understanding about unmet health care needs. However, the inequality in health care utilization cannot be resolved by personal or household effort, but requires a policy approach. Therefore, from this perspective, it is meaningful to identify the relationship between unmet dental needs of the elderly and the NHI denture coverage policy for the elderly, which was implemented to resolve inequalities due to individual payment abilities. Future studies will be needed to assess oral health policies for the elderly from a cost-effectiveness aspect, to determine whether the limited resources are used efficiently.

The objective of the present study was to identify the relation between unmet dental needs of the edentulous elderly and the NHI coverage of complete dentures that was implemented as a part of the policy to expand insurance coverage. The study used data from 2011 and 2013 CHS to analyze the edentulous elderly who were aged 75 years and older, relative to before and after 2012, when such policy was implemented. For a more accurate analysis of the effects of the NHI denture coverage policy for the elderly on unmet health care needs, recipients of the basic livelihood security benefit who qualify for the free dental prostheses program were excluded from this analysis. As a result, the study was limited to 20,400 people. Our results showed that the NHI coverage policy on dentures for the elderly did not influence unmet dental needs. The variables that had statistically significant influences were education and income levels. We determined that the elderly population with low socioeconomic status had relatively more experiences of unmet dental needs, due to the lack knowledge and socioeconomic ability to pay for dental care. Even after the NHI denture coverage policy for the elderly was implemented, the socioeconomically vulnerable population faced health issues and reduced quality of life, due to limitations in health care utilization. Therefore, policy measures should be established to resolve the issue of health inequity.

\section{References}

1. Shin Y, Shon J: The prevalence and association factors of unmet medical need: using the 1st and 2nd Korea welfare panel data. Health Soc Welf Rev 29: 111-142, 2009.

2. Lee HJ, Huh SI: Unmet health care needs and impact of type of household among the elderly in Korea. Korean J Heal Econ Policy 23: 85-108, 2017.

3. Allen SM, Mor V: The prevalence and consequences of unmet need: contrasts between older and younger adults with disability. Med Care 35: 1132-1148, 1997.

4. Yang BM, Kim JH, Lee TJ, Bae EY: Health care economics. 3rd ed. Nanam, Seoul, pp.75-125, 2015.

5. Moon J, Kang M: The prevalence and predictors of unmet medical needs among the elderly living alone in Korea: an application of the behavioral model for vulnerable populations. Health Soc Welf Rev 36: 480-510, 2016.

6. Moriya S, Miura H: Oral health and general health at the early stage of ageing: a review of contemporary studies. Jpn Dent Sci Rev 50: 15-20, 2014 https://doi.org/10.1016/j.jdsr.2013.10.002

7. Lee YH, Chun JH, Lee JH: Oral health-related quality of life improvements and satisfaction in South Korea: results from the National Health Insurance Coverage Denture Project for the Elderly. J Korean Acad Oral Health 39: 37-42, 2015. https://doi.org/10.11149/jkaoh.2015.39.1.37

8. Ahn ES, Hwang JM, Shin MS: Dental utilization associated 
factors among elderly. J Dent Hyg Sci 15: 60-66, 2015. https://doi.org/10.17135/jdhs.2015.15.1.60

9. Kim C, Paik D, Kim H, Jin B, Bae K: The oral health care needs among elderly people with daily activity problems in Yongin city. J Korean Acad Oral Health 31: 559-567, 2007.

10. Ahn E, Shin H: Awareness of dental service providers on the inclusion of denture in the coverage of the National Health Insurance. J Korean Acad Dent Adm 1: 77-88, 2013.

11. Kim YT, Choi BY, Lee KO, et al.: Overview of Korean community health survey. J Korean Med Assoc 55: 74-83, 2012. https://doi.org/10.5124/jkma.2012.55.1.74

12. Hwang BD, Choi R: The prevalence and association factors of unmet medical needs by age group in the elderly. Korean J Health Serv Manag 9: 81-93, 2015. https://doi.org/10.12811/kshsm.2015.9.1.081

13. Musacchio E, Perissinotto E, Binotto P, et al.: Tooth loss in the elderly and its association with nutritional status, socioeconomic and lifestyle factors. Acta Odontol Scand 65: 78-86, 2007. https://doi.org/10.1080/00016350601058069

14. Cano-Gutiérrez C, Borda MG, Arciniegas AJ, Borda CX: Edentulism and dental prostheses in the elderly: impact on quality of life measured with EuroQol--visual analog scale (EQ-VAS). Acta Odontol Latinoam 28: 149-155, 2015.

15. Lee SK, Park YD, Hwang KS, Kim YH: Dental technician's perception investigation about national health insurance of old person's denture in metropolitan area. J Korean Acad Dent Technol 32: 35-43, 2010.

16. Han SY, Kim CS: Recognition on national health insurance coverage and the actual condition of denture among the elderly at senior welfare centers in Seoul. J Korean Dent Assoc 50: 407-419, 2012. 